\title{
Low concentrations of commercial alcohol hand rubs facilitate growth of and secretion of extracellular proteins by multidrug-resistant strains of Acinetobacter baumannii
}

Correspondence

David W. Wareham

d.w.wareham@qmul.ac.uk

Received 11 June 2007

Accepted 3 August 2007
Justin Edwards, ${ }^{1}$ Geeta Patel ${ }^{2}$ and David W. Wareham ${ }^{1,3}$

\author{
${ }^{1}$ Department of Medical Microbiology, Division of Infection, Barts and The London NHS Trust, \\ Whitechapel, London E1 1BB, UK \\ ${ }^{2}$ Proteomics and Peptide Synthesis Facility, MRC Clinical Sciences Centre, Imperial College, \\ Hammersmith Campus, London W12 ONN, UK \\ ${ }^{3}$ Centre for Infectious Disease, Institute of Cell and Molecular Science, Barts and The London, \\ Queen Mary's School of Medicine and Dentistry, London E1 2AT, UK
}

\begin{abstract}
Acinetobacter baumannii is increasingly recognized as an important multidrug-resistant nosocomial pathogen. Recent work has highlighted enhanced growth and heightened virulence in the presence of ethyl alcohols. As alcohol-based hand rubs (ABHRs) are extensively used in health care settings, the authors set out to determine whether the hand rubs could also influence the growth of prevalent multidrug-resistant strains circulating in UK hospitals. A significant increase in growth was observed when minimal media were supplemented with concentrations of $1 \%$ and lower of four commercially available hand rubs. In addition, growth in ABHRsupplemented media resulted in secretion of proteins into the culture supernatant. One of these was identified as OmpA, which is recognized as having emulsifying activity, which could potentially confer enhanced pathogenicity to $A$. baumannii.
\end{abstract}

\section{INTRODUCTION}

Acinetobacter baumannii is a non-fermentative Gramnegative rod of the family Moraxellaciae. The organism is an opportunistic pathogen and has emerged in recent years as an important cause of ventilator-associated pneumonia, bacteraemia and sepsis in patients with burns, immunosuppression and critical illness (Joly-Guillou, 2005). Many strains also exhibit formidable antimicrobial resistance, leading to serious concerns over our ability to treat and control infection with this organism. Most of the multidrug-resistant strains circulating in UK hospitals belong to three distinct clonal groups with common PFGE profiles, namely OXA-23 clone-1, OXA-23 clone- 2 and the SouthEast clone (Coelho et al., 2006).

A. baumannii demonstrates a remarkable ability to persist in the hospital environment, with bed linen, bedside cabinets, telephone receivers, computer keyboards, sink taps, patient charts and ventilator housings all identified as environmental reservoirs (Bergogne-Berezin \& Towner, 1996). The main mechanism for transmission, however, remains spread via the hands of hospital staff. Adequate hand hygiene is therefore thought to be crucial in

Abbreviations: ABHRs, alcohol-based hand rubs; MDRAB, multidrugresistant $A$. baumannii. preventing the nosocomial transmission of A. baumannii (D'Agata et al., 2000). In many hospitals, alcohol-based hand rubs (ABHRs), consisting of varying concentrations of ethyl or isopropyl alcohol often in combination with chlorhexidine and organic emollients, have found widespread acceptance as an alternative to handwashing (Kampf \& Kramer, 2004). Recent work has shown that $A$. baumannii is able to readily metabolize low concentrations of ethyl alcohols, and, furthermore, that this leads to both enhanced growth and pathogenicity via the secretion of an unidentified substance (Smith et al., 2004). This observation is intriguing, as A. baumannii is likely to be frequently exposed to alcohol in hospital environments where a 'background level' of alcohol may exist due to the extensive use of ABHRs. Eradication of A. baumannii once established has been shown to be particularly difficult. In view of the versatile metabolic capacity of Acinetobacter species it is possible that traces of alcohol or other organic compounds in the environment could facilitate their growth and survival, perhaps even heightening their pathogenicity to humans. We therefore set out to determine whether low concentrations of commercially available ABHRs could affect the growth of prevalent strains of multidrug-resistant A. baumannii (MDRAB) and also to identify any secreted proteins which could represent virulence factors. 


\section{METHODS}

Bacterial strains and media. Three multidrug-resistant strains of $A$. baumannii belonging to the South East, OXA-23 clone-1 and OXA-23 clone-2 PFGE groups were used throughout this study. All three isolates were resistant to aminoglycosides and third-generation cephalosporins (MDRAB) and both of the OXA-23 strains were also resistant to carbapenems (MDRAB-C). Strains were grown in either the enriched medium YPAD containing $1 \%$ yeast extract, $2 \%$ Bacto peptone, $2 \%$ glucose and $300 \mu \mathrm{M}$ adenine or in M9 Minimal Media (MM) consisting of $1 \times \mathrm{M} 9$ salts $\left(0.6 \% \mathrm{NaHPO}_{4}, 0.3 \% \mathrm{KH}_{2} \mathrm{PO}_{4}\right.$,

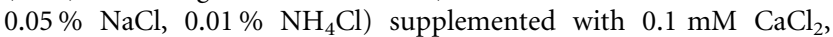
$0.002 \mathrm{M} \mathrm{MgSO}_{4}$ and $0.4 \%$ glucose.

Growth analyses. Stationary phase cultures were made by incubation of each strain overnight at $37^{\circ} \mathrm{C}$ in $5 \mathrm{ml}$ YPAD. One hundred microlitres of this culture was used to inoculate $10 \mathrm{ml}$ of both YPAD and MM with the addition of $0.1-10 \%(\mathrm{v} / \mathrm{v})$ of either $100 \%$ ethanol or the commercially available ABHRs Skinman, Softalind, Spirigel and Purell. The cultures were reincubated and growth was quantified spectrophotometrically by recording the $\mathrm{OD}_{600}$ at set time intervals. All readings were blanked versus uninoculated media to account for any turbidity resulting from the addition of ethanol or ABHR to the media. Measurements were taken approximately every hour for 6-8 $\mathrm{h}$ in experiments conducted using YPAD with final measurements taken overnight. In experiments performed using MM, measurements were taken twice daily for $48 \mathrm{~h}$. Incubation of each strain was also performed, at the same concentrations, following supplementation of the media with the non-alcohol-based hand rub Clinell.

Viable counts. To confirm that any observed increase in $\mathrm{OD}_{600}$ was due to an increase in the number of viable bacteria, the number of c.f.u. $\mathrm{ml}^{-1}$ was determined as follows. For each time point, serial dilutions of $100 \mu \mathrm{l}$ of culture were made down to a concentration of $10^{-9}$. Counts were performed following overnight incubation of $100 \mu$ aliquots of each dilution spread onto LB agar plates.

Analysis of extracellular protein secretion. Proteins secreted following growth in MM with and without ABHR supplementation were prepared as follows. Cells from $1.5 \mathrm{ml}$ of stationary phase culture were pelleted by centrifugation at $16000 \mathrm{~g}$ for $4 \mathrm{~min}$. Supernatant $(1 \mathrm{ml})$ was transferred to a fresh tube and proteins were precipitated by addition of $350 \mu \mathrm{l} 50 \%(\mathrm{w} / \mathrm{v})$ trichloroacetic acid. After incubation on ice for $30 \mathrm{~min}$, precipitated protein was collected by centrifugation at $16000 \mathrm{~g}$ for $10 \mathrm{~min}$. Pellets were washed with $1 \mathrm{ml}$ acetone and resuspended in $15 \mu \mathrm{l}$ NuPAGE lithium dodecyl sulphate sample buffer. SDS-PAGE analysis was carried out using XCell SureLock Mini-Cell (Invitrogen) apparatus. Samples were heated at $70{ }^{\circ} \mathrm{C}$ for $10 \mathrm{~min}$ before loading onto NuPAGE Novex 4$12 \%$ (v/v) Pre-Cast Bis-Tris gels and electrophoresed at $200 \mathrm{~V}$ for 35$50 \mathrm{~min}$ in NuPAGE MOPS SDS Running Buffer. Gels were removed from the apparatus, washed three times with $100 \mathrm{ml}$ deionized $\mathrm{H}_{2} \mathrm{O}$ and then stained for $1 \mathrm{~h}$ with $20 \mathrm{ml}$ SimplyBlue SafeStain (Invitrogen). Clear backgrounds for photography were obtained by further washing with deionized $\mathrm{H}_{2} \mathrm{O}$ for $1-3 \mathrm{~h}$. Molecular mass was determined by comparison with prestained standards (New England Biolabs).

Proteomics analysis. Protein bands labelled 1 and 2 (Fig. 4) were excised from the gels, destained, reduced and alkylated prior to in-gel digestion with trypsin. Briefly, the gel pieces were destained with $50 \mathrm{mM}$ ammonium bicarbonate $/ 50 \%$ acetonitrile, reduced with dithiothreitol $(10 \mathrm{mM}$ in $100 \mathrm{mM}$ ammonium bicarbonate for $30 \mathrm{~min}$ ) and alkylated with iodoacetamide $(55 \mathrm{mM}$ in $100 \mathrm{mM}$ ammonium bicarbonate for $20 \mathrm{~min}$ ). Following a wash step with $100 \mathrm{mM}$ ammonium bicarbonate and dehydration with acetonitrile, the gel pieces were digested with $25 \mu \mathrm{l} 6 \mathrm{ng}$ trypsin $\mu^{-1}$ in $50 \mathrm{mM}$ ammonium bicarbonate for $5 \mathrm{~h}$ at $40{ }^{\circ} \mathrm{C}$. The tryptic peptide fragments were extracted twice: firstly with $2 \%$ acetonitrile/ $1 \%$ formic acid for $30 \mathrm{~min}$ and then with $50 \%$ acetonitrile/50\% aqueous $1 \%$ formic acid for another $30 \mathrm{~min}$. All treatments were performed robotically using a MassPrep Workstation (Micromass). All extracts from a given gel piece were combined, reduced to $1-2 \mu \mathrm{l}$ in a Speed$\mathrm{Vac}$ concentrator and reconstituted in $0.1 \%$ formic acid for mass spectral analysis by LC-MS/MS using the CapLC/Q-ToF2 (Micromass). Samples were transferred to the autosampler of a CapLC and eluted through a reverse-phase capillary column directly into the nano-electrospray ion source of a Q-ToF2 mass spectrometer. The instrument was operated in survey scanmode, with the range $m / z$ 400-1800. All fragment ion (MS/MS) spectra from the sample were searched using the MASCOT search tool (Matrix Science) using the MS protein sequence database to obtain protein identification. MASCOT scores only for tryptic peptide fragments that were above the significance level indicating identity or extensive homology were reported.

\section{RESULTS}

\section{Effect of ethanol and ABHR supplementation on the growth of MDRAB}

All three strains of A. baumannii were able to grow in YPAD supplemented with ethanol at $1 \%$ or less. Growth in YPAD supplemented with ethanol at $10 \%$ was not supported. There was no significant difference in growth rate between supplemented and unsupplemented YPAD media. Supplementation at $0.1 \%$ or less did, however, result in growth to a higher final $\mathrm{OD}_{600}$ for all strains (Fig. 1).

When strains were grown in MM supplemented with $1 \%$ ethanol or less, both a significantly faster initial rate of growth and growth to a higher final $\mathrm{OD}_{600}$ were observed, with $1 \%$ ethanol being the optimal concentration. All strains failed to grow in $\mathrm{MM}+10 \%$ ethanol (Fig. 2).

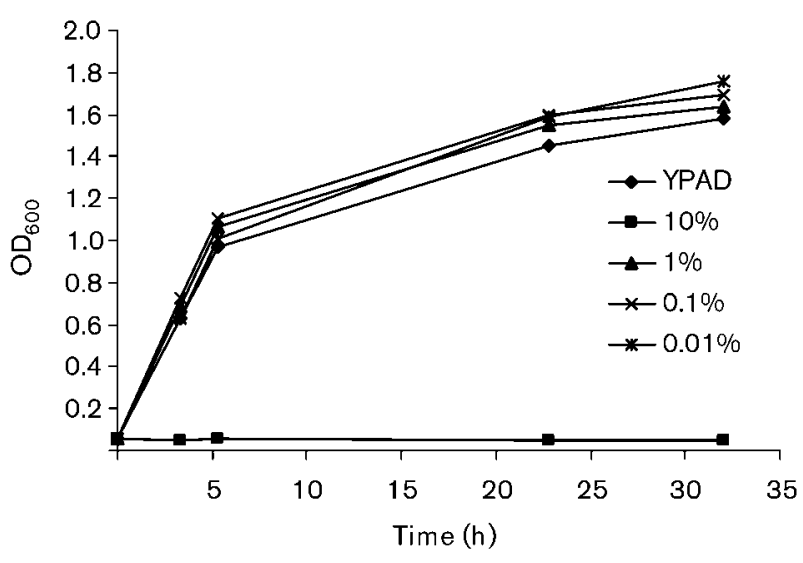

Fig. 1. Growth of MDRAB in enriched media following supplementation with $0.01-10 \%$ ethanol. 


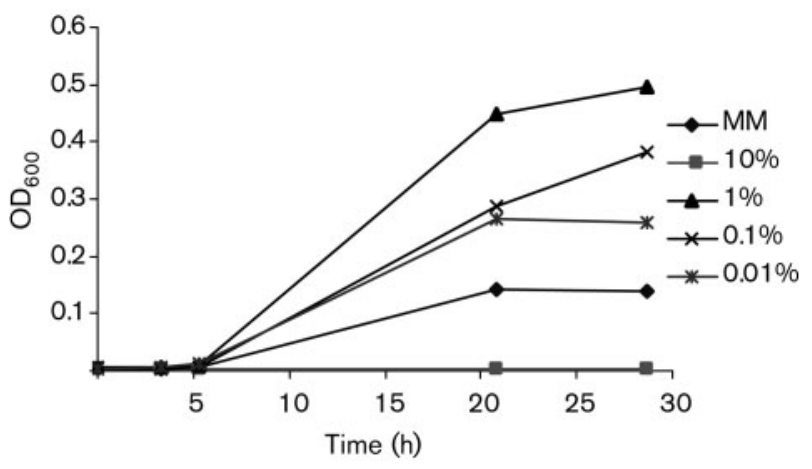

Fig. 2. Growth of MDRAB in minimal media following supplementation with $0.01-10 \%$ ethanol.

Growth of all strains in YPAD media supplemented with $0.1 \%(\mathrm{v} / \mathrm{v})$ of both Softalind and Skinman ABHRs resulted in a small but insignificant rise in $\mathrm{OD}_{600}$. When $\mathrm{MM}$ was used as the growth medium, growth enhancement was observed for all strains following supplementation with $1 \%, 0.1 \%$ and $0.01 \%$ of each ABHR. Optimal growth occurred at $1 \%$ supplementation although growth was initially faster at $0.1 \%$. Strains were unable to grow at $10 \%$ supplementation or when supplemented with Clinell. The same pattern was seen in all strains when incubated with all four ABHRs (Fig. 3). Increases in $\mathrm{OD}_{600}$ were also found to correlate with an increase in the number of viable bacteria, as measured by colony counts, regardless of the growth media used or supplementation with ethanol or ABHRs.

\section{Effect of ABHRs on secretion of extracellular proteins}

Proteins present in culture supernatants of all three strains of MDRAB grown in MM supplemented with $1 \%$ of each ABHR were examined by SDS-PAGE. A protein of between 17 and $20 \mathrm{kDa}$ was detected in experiments performed

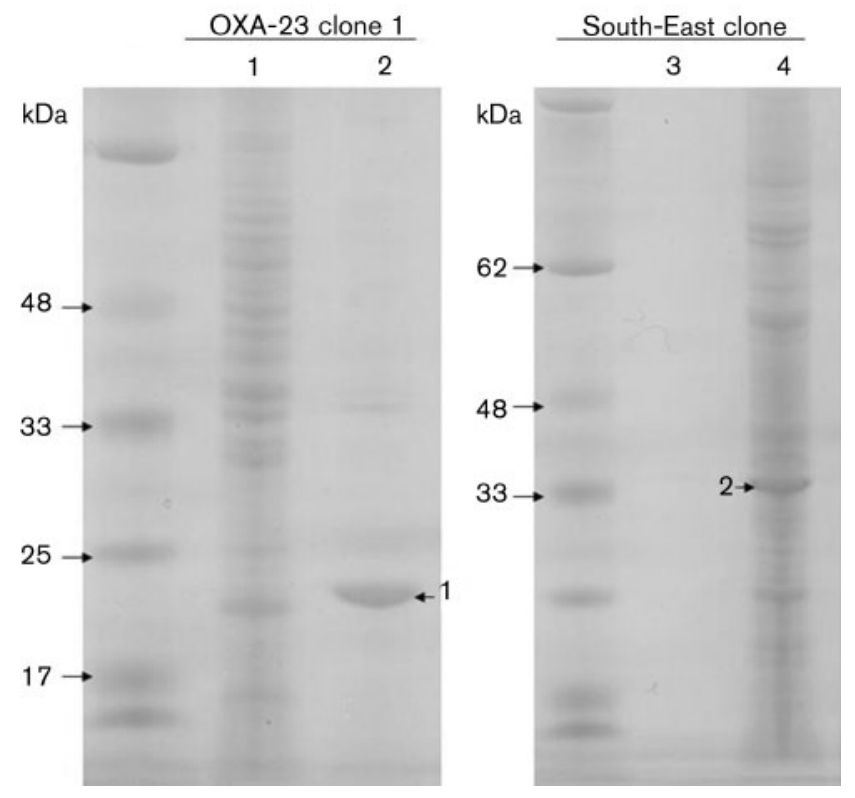

Fig. 4. Secretion of extracellular proteins by MDRAB following growth in MM supplemented with ABHRs. Lanes: 1, MM alone; 2, $\mathrm{MM}+1 \%$ Softalind; $3, \mathrm{MM}$ alone; $4, \mathrm{MM}+1 \%$ Skinman. Protein bands labelled 1 and 2 were excised from the gel and examined by Q-ToF MS/MS analysis.

using OXA-23 clone-1 and OXA-23 clone 2 whilst a protein of approximately $33 \mathrm{kDa}$ was produced by the South-East clone (Fig. 4)

\section{Identification of secreted proteins by MS}

Bands labelled 1 and 2 (Fig. 4), containing the proteins, were cut from the gel and subjected to proteomics analysis for protein identification. Tryptic peptide fragments were analysed by a Q-TOF2 mass spectrometer and the mass spectral data were used to search protein databases by the

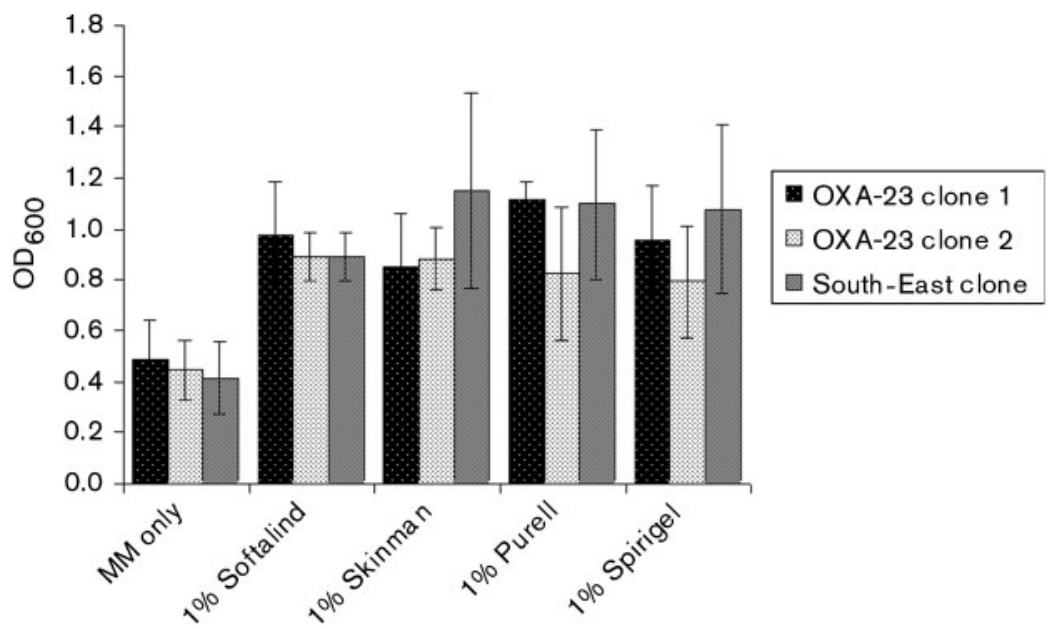

Fig. 3. Growth of MDRAB in minimal media supplemented with $1 \%$ ABHR. All three strains of MDRAB grew to a higher $\mathrm{OD}_{600}$ after $48 \mathrm{~h}$ following supplementation with $1 \%$ Softalind, Skinman, Purell or Spirigel ABHRs ( $P<0.05$, paired Student's $t$-test). 
Table 1. Identification of secreted proteins from MDRAB following growth in minimal media supplemented with $1 \% \mathrm{ABHR}$

\begin{tabular}{|llcccc|}
\hline $\begin{array}{l}\text { Protein } \\
\text { no. }\end{array}$ & Identification & MASCOT score & $\begin{array}{c}\text { Peptides matched/ } \\
\text { protein coverage }\end{array}$ & $\begin{array}{c}\text { Theoretical molecular } \\
\text { mass }\end{array}$ & $\begin{array}{c}\text { Actual molecular } \\
\text { mass }\end{array}$ \\
\hline 1 & $\begin{array}{c}\text { Hypothetical protein from } \\
\text { Acinetobacter ADP-1 } \\
\text { Outer-membrane protein A - } \\
\text { A. baumannii }\end{array}$ & 117 & $1 / 7 \%$ & $26.1 \mathrm{kDa}$ & $22 \mathrm{kDa}$ \\
& 922 & $14 / 44 \%$ & $38.4 \mathrm{kDa}$ & $34 \mathrm{kDa}$ \\
\hline
\end{tabular}

MASCOT search tool. Based on a high probability MOWSE score, the protein secreted by the South-East clone was identified as outer-membrane protein $\mathrm{A}(\mathrm{OmpA})$ from $A$. baumannii and the proteins secreted by OXA-23 clones 1 and 2 were identified as a hypothetical protein from $A$. baumannii ADP-1. The number of tryptic peptide fragments matched, together with protein coverage and putative MOWSE scores by MASCOT, are summarized in Table 1. There was good correlation between the theoretical and actual masses of the proteins identified. Although the MOWSE score for band 1 was low, this reflects poor quality data rather than an incorrect match. The high score for band 2 indicates a highly significant hit for the protein.

\section{DISCUSSION}

Previous work has demonstrated that low concentrations of ethanol enhance both the growth and virulence of Acinetobacter spp. The results of our study indicate that the growth of multidrug-resistant strains of A. baumannii is also enhanced when exposed not only to ethanol but also to ABHRs. This phenomenon is most apparent when MDRAB is incubated in a minimal growth medium as, presumably, the addition of ethanol or ABHR provides an additional carbon source which is readily utilized. All four ABHRs tested, Skinman, Softalind, Spirigel and Purell, were able to significantly enhance MDRAB growth at $1 \%$ or less. In NHS hospitals, these rubs are either wallmounted or placed next to a patient's bedside. The solutions are usually pump-dispensed directly onto the hands, which may result in considerable spillage and contamination of the surrounding environment. In contrast to the ABHRs tested, the non-alcohol-based product Clinell, which relies on the biocidal activity of two cationic surfactants, did not support the growth of MDRAB at all. If low levels of ABHRs do indeed exist in clinical areas where they are intensively used, this could affect the survival and persistence of MDRAB.

Our results also indicate that there are distinct differences in the extracellular protein profiles when MDRAB is grown in $\mathrm{MM}$ or $\mathrm{MM}$ supplemented with ABHRs. Exposure of the OXA-23 clones 1 and 2 to ABHRs resulted in the secretion of a protein whose function is yet to be identified; however, the identification of OmpA as the protein in the supernatant of the South-East clone is particularly interesting. OmpA possesses emulsifying activity, which could be useful in scavenging carbon for growth from complex hydrocarbon energy sources. The secretion of OmpA by Acinetobacter following exposure to $0.5 \%$ ethanol in minimal media has also been described by Walzer et al. (2006), suggesting that it may be an important response to growth and survival under low-nutrient conditions. As well as conveying a growth advantage, bioemulsifiers may also play an important role in bacterial adhesion, quorum sensing and the development of biofilms (Ron \& Rosenberg, 2001). Direct evidence for a role of $A$. baumannii outer-membrane proteins in pathogenesis comes from work by Choi et al. (2005), whereby purified outer-membrane protein 38 (Omp38) was able to induce apoptosis in respiratory epithelial cells. The highly clonal, hospital-acquired, multidrug-resistant strains of $A$. baumannii circulating in the UK appear to be less virulent than drug-sensitive community strains (Leung et al., 2006). Therefore, any enhanced effect on virulence due to ABHR exposure could have major clinical implications.

This study presents some interesting results which could have significant implications for infection control practices in those hospitals experiencing outbreaks of MDRAB. Concerns over the use of ABHRs have already been expressed with respect to their lack of activity against the spores that potentiate Clostridium difficile infection. In light of this study, the widespread use of ABHRs, in areas where A. baumannii infection is seen as a particular concern, may also need to be reassessed. Further epidemiological studies are needed to assess the impact of widespread use of ABHRs for the control of MDRAB.

\section{ACKNOWLEDGEMENTS}

We wish to thank Dr J. Turton and Dr T. Pitt for supplying strains of A. baumannii from the Epidemiological Typing Reference Unit at The Health Protection Agency (Colindale, UK). This work was supported by a Hospital Infection Society Small Research Grant awarded to Dr D. Wareham. Sample preparation and mass spectral analyses were provided by Justin Lock and Dinah Rahman (MRC - Clinical Sciences Centre, London).

\section{REFERENCES}

Bergogne-Berezin, E. \& Towner, K. J. (1996). Acinetobacter spp. as nosocomial pathogens: microbiological, clinical, and epidemiological features. Clin Microbiol Rev 9, 148-165. 
Choi, C. H., Lee, E. Y., Lee, Y. C., Park, T. I., Kim, H. J., Hyun, S. H., Kim, S. A., Lee, S. K. \& Lee, J. C. (2005). Outer membrane protein 38 of Acinetobacter baumannii localizes to the mitochondria and induces apoptosis of epithelial cells. Cell Microbiol 7, 1127-1138.

Coelho, J. M., Turton, J. F., Kaufmann, M. E., Glover, J., Woodford, N., Warner, M., Palepou, M. F., Pike, R., Pitt, T. L. \& other authors (2006). Occurrence of carbapenem resistant Acinetobacter baumannii clones at multiple hospitals in London and South East England. J Clin Microbiol 44, 3623-3627.

D'Agata, E. M. C., Thayer, V. \& Schaffner, W. (2000). An outbreak of Acinetobacter baumannii: the importance of cross-transmission. Infect Control Hosp Epidemiol 21, 588-591.

Joly-Guillou, M. L. (2005). Clinical impact and pathogenicity of Acinetobacter. Clin Microbiol Infect 11, 868-873.
Kampf, G. \& Kramer, A. (2004). Epidemiological background of hand hygiene and evaluation of the most important agents for scrubs and rubs. Clin Microbiol Rev 17, 863-893.

Leung, W.-S., Chu, C.-M., Tsang, K.-Y., Lo, F.-H., Lo, K.-F. \& Ho, P.-L. (2006). Fulminant community-acquired Acnetobacter baumannii pneumonia as a distinct clinical syndrome. Chest 129, 102-109.

Ron, E. Z. \& Rosenberg, E. (2001). Natural roles of biosurfactants. Environ Microbiol 3, 229-236.

Smith, M. G., Des Etages, S. G. \& Snyder, M. (2004). Microbial synergy via an ethanol-triggered pathway. Mol Cell Biol 24, 3874-3884.

Walzer, G. E., Rosenberg, E. \& Ron, E. Z. (2006). The Acinetobacter outer membrane protein A (OmpA) is a secreted emulsifier. Environ Microbiol 8, 1026-1032. 